\title{
Real-Time Estimation of Hip Range of Motion for Total Hip Replacement Surgery
}

\author{
Yasuhiro Kawasaki ${ }^{1}$, Fumihiko Ino ${ }^{1}$, Yoshinobu Sato $^{2}$, Nobuhiko Sugano ${ }^{2}$, \\ Hideki Yoshikawa $^{2}$, Shinichi Tamura ${ }^{2}$, and Kenichi Hagihara ${ }^{1}$ \\ 1 Graduate School of Information Science and Technology \\ ${ }^{2}$ Graduate School of Medicine \\ Osaka University \\ y-kawask@ist.osaka-u.ac.jp
}

\begin{abstract}
This paper presents the design and implementation of a range of motion estimation method that is capable of fine-grained estimation during total hip replacement (THR) surgery. Our method combines an adaptive refinement strategy with a high performance computing system in order to enable real-time estimation. The experimental results indicate that the implementation on a cluster of 64 PCs enables intraoperative estimation of $360 \times 360 \times 180$ stance configurations within a half minute, and thereby plays a key role in selecting and aligning the optimal combination of artificial joint components during THR surgery.
\end{abstract}

\section{Introduction}

Total hip replacement (THR) [1,2] is a surgical procedure that relieves patients of hip pain and removes their difficulty in walking by replacing the hip joint with an artificial joint. The key issue in this surgery is to select and align the optimal combination of artificial joint components: the cup, head, neck, and stem components as illustrated in Fig. 1(a) These optimal selection and alignment are important for both the surgeon and patient because either inappropriate or malpositioned components increase the risk of clinical problems such as dislocation, wear, and loosening [2,3].

In order to assist the surgeon in the selection and alignment, range of motion (ROM) estimation systems [1, 2, 4, 3, 5] have been developed in the past. Earlier systems are useful in developing preoperative surgical plans because they present the limitation of hip movement on three-dimensional (3-D) polygonal surface models reconstructed from patients' computed tomography (CT) images. However, the preoperative plans may need to be changed by unexpected conditions that reveal during surgery. For example, if the bone tissue around the preoperatively planned position is known to be fragile, another position has to be selected according to intraoperative circumstances. Thus, intraoperative estimation is essential to overcome such unexpected conditions.

One issue to develop intraoperative estimation systems is a large amount of computation due to collision detections (CDs) required for ROM estimation. For example, an earlier system [4] on a Sun Ultra 30 running at $300 \mathrm{MHz}$ takes 0.05 seconds for detecting a collision for a stance configuration, so that it takes approximately 13 days when computing a 3 -D ROM with $360 \times 180 \times 360=23328000$ configurations: $360^{\circ}$, $180^{\circ}$, and $360^{\circ}$ for yaw $(\phi)$, pitch $(\theta)$, and role $(\psi)$ angles, respectively, as illustrated 


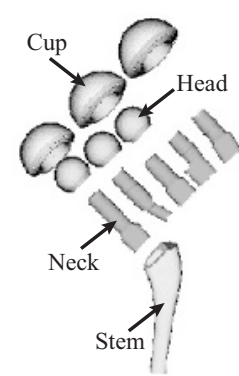

(a)

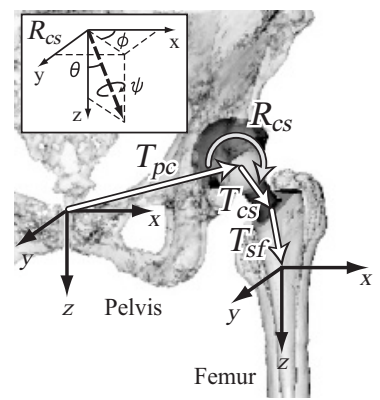

(b)

Fig. 1. (a) Components of artificial joint and (b) representation of hip joint motion.

in Fig. 1(b), Therefore, to achieve real-time estimation during surgery, earlier systems need to degrade the quality of estimations by limiting the area of estimations [1,2] and by degrading the number of stance configurations [3] or the resolution of images [5].

In contrast to these coarse-grained estimations, the key contribution of this paper is to enable fine-grained estimations during surgery. To enable this, we have developed a real-time estimation system based on two key methods: 1) adaptive ROM refinement for computational complexity reduction and 2) parallelization for further acceleration. Our results indicate that the combination of the two methods is 11 times faster than a previous parallel system [6] without adaptive refinement.

The remainder of the paper is organized as follows. Section 2 summarizes an overview of ROM estimation while Section 3 gives the design and implementation of our method. Section 4 presents some experimental results. Section 5 concludes this paper.

\section{Background: Range of Motion (ROM) Estimation}

To describe the details of ROM estimation, we first show a brief representation of hip joint motion described in [3]. Let $M_{p f}$ denote the transformation from the pelvis coordinate system (pelvis-CS) to the femur coordinate system (femur-CS). As illustrated in Fig. 1(b), the hip joint motion is given by:

$$
M_{p f}=T_{p c} T_{c s} R_{c s} T_{s f}
$$

where $T_{p c}$ is a $4 \times 4$ transformation matrix representing the orientation of the cup in the pelvis-CS, $T_{c S}$ is a fixed transformation matrix determined by the selected head and neck components, $R_{c s}$ is a variable transformation matrix constrained to the rotational motion, and $T_{s f}$ is a transformation matrix representing the reverse orientation of the stem in the femur-CS. Both $T_{p c}$ and $T_{s f}$ are determined by one of the following two methodologies. For preoperative assistances, the surgeon determines them by visualization and experience. For intraoperative assistances, optical 3-D position sensors give the 


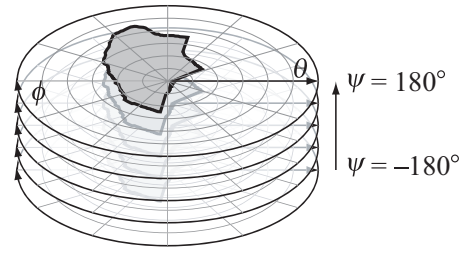

(a)

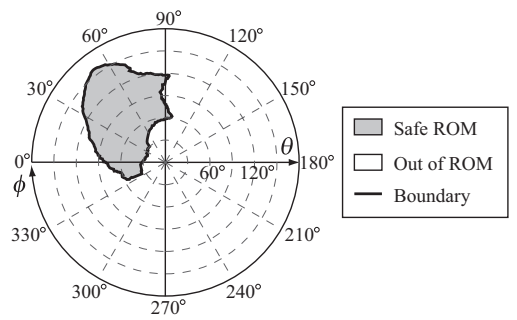

(b)

Fig. 2. (a) A 3-D ROM and (b) its slice, where $\psi=0^{\circ}$, given in polar coordinates. The entire region in the slice is separated into two pieces by a closed line. Enclosed region represents a safe ROM while the outside region represents an out of the ROM.

actual values of $T_{p c}$ and $T_{s f}$ by measuring implanted components. Thus, intraoperative estimations based on measured $T_{p c}$ and $T_{s f}$ are required to obtain precise ROMs.

Given $T_{p c}, T_{c s}$, and $T_{s f}$, the safe ROM is defined as a set of rotation transformation matrices, $\mathcal{S}$, such that for all $R_{c s} \in \mathcal{S}, R_{c s}$ avoids any implant-implant, bone-implant, and bone-bone impingements. Fig. 2 shows an example of the safe ROM with two features: a closed line shapes the boundary between inside and outside the ROM, and the safe ROM does not always include the origin. Therefore, ROM estimation is a search problem that locates the boundary in a 3-D space. Since $R_{c s}$ is defined in a 3-D space, ROM estimation is a compute-intensive application. In the following, we represent $R_{c s}$ by the Euler angles, $(\phi, \theta, \psi)\left(0^{\circ} \leq \phi<360^{\circ}, 0^{\circ} \leq \theta<180^{\circ},-180^{\circ} \leq \psi<180^{\circ}\right)$, as shown in Fig. 1(b)

To compute $\mathcal{S}$, earlier systems investigate every stance configuration, $(\phi, \theta, \psi)$, sampled at uniform intervals, whether it causes impingements. Therefore, the amount of computation increases with the number of stance configurations. Furthermore, it depends on the complexity of the $\mathrm{CD}$ algorithm employed for each stance configuration.

\section{Real-Time ROM Estimation}

This section describes the two key methods employed in our system.

\subsection{Adaptive ROM Refinement}

The key idea of adaptive ROM refinement, which aims to reduce the amount of computation, is to investigate in detail the stance configurations only close to the boundary between inside and outside the ROM. To realize this non-uniform refinement, our estimation algorithm employs the following two techniques.

1. Hierarchical refinement of sampling intervals.

2. Status control of each stance configuration. 


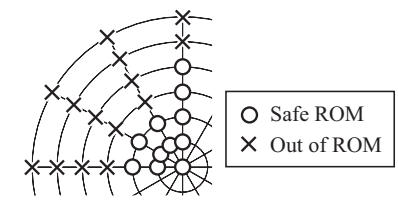

(a)

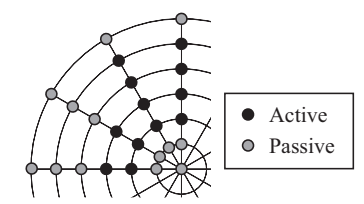

(b)

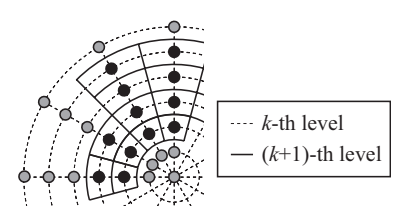

(c)

Fig. 3. Adaptive ROM refinement. (a) Given a safe ROM at the $k$-th level, (b) Eq. (2) determines the status of each stance configuration, and then (c) the algorithm refines the sampling intervals only around active configurations before advancing to the next $(k+1)$-th level.

Let $i_{k}$ be the sampling interval for stance configurations at the $k$-th level of estimation hierarchy, where $k \geq 1$. To compute the safe ROM in a coarse-to-fine manner, the algorithm refines the interval when advancing the estimation level as follows: $i_{k+1}=$ $i_{k} / 2$, where $i_{k} \geq 1^{\circ}$.

At each level, the algorithm associates each stance configuration with a status, $\mathcal{C}_{\phi, \theta, \psi} \in\{$ active, passive $\}$. Then, it refines the sampling intervals only around active configurations and investigates the refined configurations whether they cause impingements. Refinement around the remaining passive configurations is omitted in order to reduce the amount of computation.

To enable this omission, the algorithm assumes that a stance configuration surrounded by others with impingements (or no impingements) also causes impingements (or no impingements). Based on this assumption, such surrounded configurations can be omitted. The status of each stance configuration is given by:

$$
\mathcal{C}_{\phi, \theta, \psi}=\left\{\begin{array}{cc}
\text { passive, } & \text { if } \forall p, q, r \in\{1,-1\} \mid(\phi+p, \theta+q, \psi+r) \in \mathcal{S} \\
& \vee \forall p, q, r \in\{1,-1\} \mid(\phi+p, \theta+q, \psi+r) \notin \mathcal{S}, \\
\text { active, } & \text { otherwise. }
\end{array}\right.
$$

In addition to the computational complexity reduction, our adaptive algorithm has another advantage compared to earlier algorithms without adaptive refinement. Since the algorithm refines the sampling intervals as it moves up the hierarchy, it enables progressive visualization of the safe ROM. Therefore, an outline of the safe ROM is visible in the early phase of estimation, allowing surgeons to immediately terminate the ongoing estimation when the outline is known to be obviously an unoptimal result.

\subsection{Parallelization}

In the presented algorithm, a CD for stance configuration $(\phi, \theta, \psi)$ is independent of that for another configuration $\left(\phi^{\prime}, \theta^{\prime}, \psi^{\prime}\right)$ if and only if status $\mathcal{C}_{\phi, \theta, \psi}$ determines whether to sample $\left(\phi^{\prime}, \theta^{\prime}, \psi^{\prime}\right)$ or not at the refinement. That is, any two configurations without causal relation between them can be processed in parallel. Therefore, our method exploits task-level parallelism of the ROM estimation. Here, a task corresponds to CDs for a set of configurations in the same level and for that of configurations in different levels but with no causal relation among the configurations. 


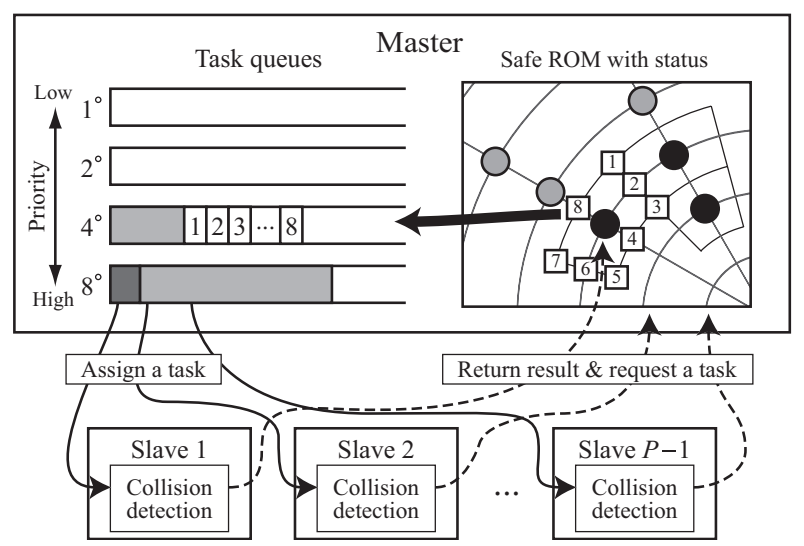

Fig. 4. Adaptive ROM estimation based on master/slave paradigm. Given $P$ nodes, $P-1$ slaves detect collisions for tasks assigned from a master while the master manages estimation results and task queues for each estimation level, and samples tasks $(1,2, \ldots, 8)$ according to Eq. [2].

In order to exploit this parallelism, our method employs the master/slave (M/S) paradigm, where computing nodes are classified into two groups: a master and the remaining slaves. Fig. 4 shows an overview of the method. While the slaves detect collisions for tasks assigned from the master, the master manages estimation results, namely the safe ROM, and updates the status of each configuration. According to this status, the master determines which region need to be refined, and then samples stance configurations for the next level. These newly sampled configurations are enqueued as tasks to a queue prepared for each estimation level. Tasks are dequeued when assigning them to slaves. Since idle slaves are selected for this assignment, the M/S paradigm is capable of dynamic load balancing among slaves.

Note here that our method prevents synchronization among slaves when advancing the estimation level. This asynchronous behavior gives higher speedup but allows the master to simultaneously queue tasks sampled from different levels. Such tasks should be processed from the coarse-grained level because tasks in this level can generate many tasks due to the refinement. Therefore, each queue in the master has a priority to enable this coarse-to-fine processing.

\section{Results}

In this section, we evaluate the performance of our adaptive method by comparing it with the previous non-adaptive method [6] on a cluster of PCs. The cluster consists of 64 symmetric multiprocessor (SMP) nodes, each with two Pentium III 1-GHz CPUs and a Myrinet [7] and Fast Ethernet interconnects that provide bandwidth of $2 \mathrm{~Gb} / \mathrm{s}$ and 100 $\mathrm{Mb} / \mathrm{s}$, respectively.

We have implemented our method using the $\mathrm{C}++$ language and the MPICH-SCore library [8], a highly portable and efficient implementation of the Message Passing Interface (MPI) standard [9]. For each of CDs, we used the V-COLLIDE library [10 [1], which rapidly detects precise collisions at $O(n+m)$ time, where $n$ and $m$ denote the 
Table 1. Execution time and its breakdown for fine-grained ROM estimation with $360 \times 180 \times$ 360 stance configurations. AR denotes adaptive refinement.

\begin{tabular}{|c|c|c|c|c|c|c|}
\hline \multirow{4}{*}{ Breakdown } & \multicolumn{6}{|c|}{ Execution time (second) } \\
\hline & \multirow{2}{*}{\multicolumn{2}{|c|}{ Sequential }} & \multicolumn{4}{|c|}{ Parallel on 128 CPUs } \\
\hline & & & \multicolumn{2}{|c|}{ Fast Ethernet } & \multicolumn{2}{|c|}{ Myrinet } \\
\hline & w/o AR & w/ AR & w/o AR & w/ AR & w/o AR & w/ AR \\
\hline Initialization of V-COLLIDE & \multicolumn{6}{|c|}{6.0} \\
\hline Estimation at level $1\left(i_{1}=16^{\circ}\right)$ & - & 6 & - & 0.1 & - & 0.1 \\
\hline level $2\left(i_{2}=8^{\circ}\right)$ & - & 10 & - & 0.1 & - & 0.1 \\
\hline level $3\left(i_{3}=4^{\circ}\right)$ & - & 43 & - & 0.8 & - & 0.5 \\
\hline level $4\left(i_{4}=2^{\circ}\right)$ & - & 202 & - & 3.1 & - & 2.3 \\
\hline level $5\left(i_{5}=1^{\circ}\right)$ & 16318 & 996 & 191 & 16.8 & 135 & 10.7 \\
\hline Total & 16324 & 1263 & 197 & 26.8 & 141 & 19.6 \\
\hline
\end{tabular}

number of polygonal objects and that of polygonal objects very close to each other, respectively.

The datasets of the pelvis and femur were composed of 116270 and 30821 polygons, respectively. See [3, 12, 6] for the detail explanation of how we generated them and utilized the system during surgery. The estimation hierarchy was composed of five levels with $i_{1}=16^{\circ}$. The grain size of a task, namely the number of configurations that compose a task, was experimentally selected: 50 and 10000 configurations for the adaptive and non-adaptive methods, respectively.

Table 1 shows the execution time for fine-grained ROM estimation with $360 \times 180$ $\times 360$ stance configurations. Compared to the parallel non-adaptive method, the parallel adaptive method reduces the execution time on Myrinet from 141 to 19.6 seconds and that on Fast Ethernet from 197 to 26.8 seconds. These timing results, below a half minute, are acceptable for intraoperative surgical planning. Since the sequential adaptive method takes 1263 seconds, parallelization as well as adaptive refinement is also a key method for intraoperative assistances.

In addition to this timing benefit, the adaptive method enables progressive visualization, as we mentioned in Section 3.1. For example, after 6.1, 6.1, 6.6, and 8.9 seconds on Myrinet, the system outlined the safe ROM with sampling intervals of $16^{\circ}, 8^{\circ}, 4^{\circ}$, and $2^{\circ}$, respectively. Here, even the initial coarsest level took 6.1 seconds. This is due to the initialization of V-COLLIDE, which takes 6 seconds to construct data structure from the input polygons for rapid CD. Thus, although V-COLLIDE requires pre-processing time for rapid CD, we think that progressive visualization is essential for intraoperative applications to report the progress of processing to surgeons.

Fig.5explains how the adaptive method reduces the amount of computation. It shows distribution maps of sampling intervals for the adaptive and non-adaptive methods. We can see that the adaptive method investigates in detail the stance configurations only around the boundary of the ROM. This reduces the number of investigated configurations from 23328000 to 1594816 configurations, and thereby the speedup to the non-adaptive method reaches a factor of $12.9(=16$ 324/1263) on a single CPU machine.

Finally, to evaluate the scalability of our method, we measured its parallel speedup on different numbers of CPUs (Fig. 6). Here, the parallel speedup is the ratio of the sequential execution time to the parallel execution time. While the non-adaptive method 


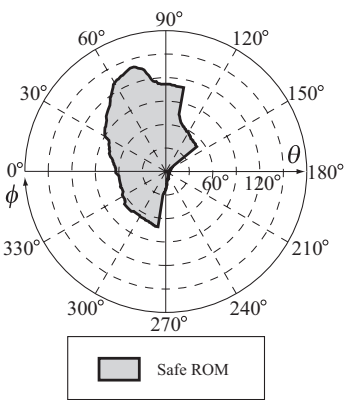

(a)

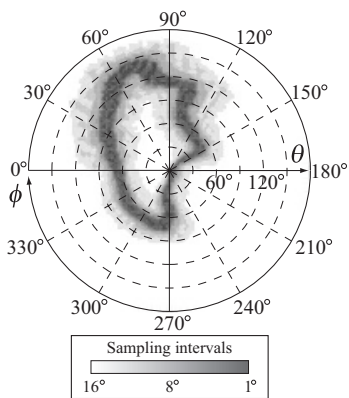

(b)

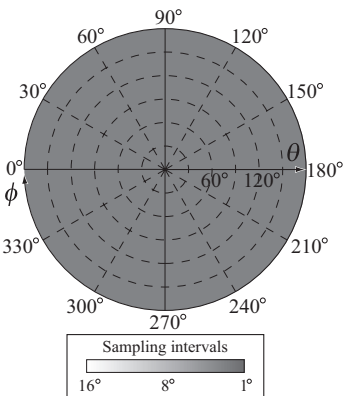

(c)

Fig. 5. (a) A slice of computed safe ROM, where $\psi=0^{\circ}$, and distribution maps of sampling intervals (b) for adaptive method and (c) for non-adaptive method.

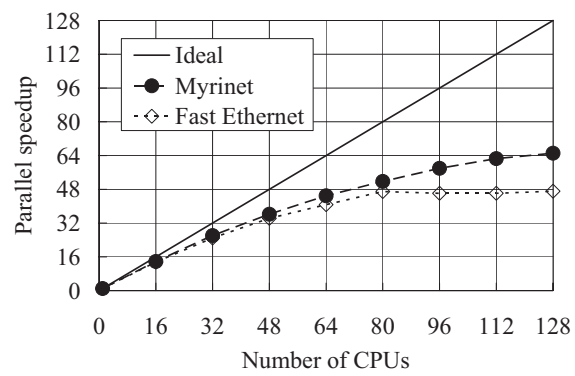

(a)

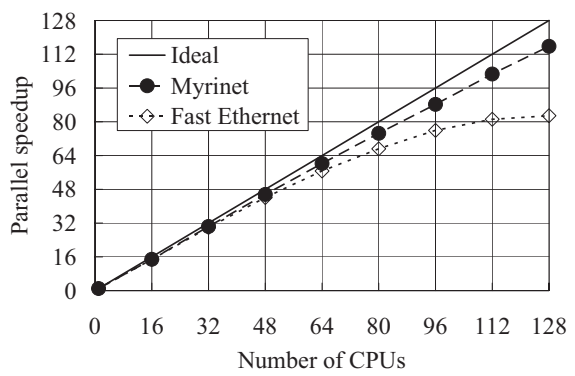

(b)

Fig. 6. (a) Parallel speedup of adaptive method and (b) that of non-adaptive method.

achieves linear speedup, the adaptive method seems to reach the peak performance as the number of CPUs increases. This is mainly due to the less parallelism of the adaptive method compared to that of the non-adaptive method. That is, although the adaptive method reduces the amount of computation, it loses the complete independence of tasks that the non-adaptive method has. Furthermore, the adaptive method needs the master to compute the status for each stance configuration. Thus, less parallelism and additional work decrease the parallel speedup of the adaptive method.

Note here that although the non-adaptive method offers better parallel speedup than the adaptive method, it takes approximately seven times longer execution time on 128 CPUs. Therefore, parallel speedup is not always a good measure for the evaluation of parallel methods. 


\section{Conclusions}

We have presented a ROM estimation method that is capable of intraoperative estimation for THR surgery. Our method achieves real-time estimation based on two methods: adaptive ROM refinement strategy where the safe ROM is non-uniformly refined in a coarse-to-fine manner to reduce the computational complexity; and HPC approach where the safe ROM is computed in parallel to obtain further acceleration.

The experimental results indicate that the implementation on a cluster of 64 PCs enables intraoperative estimation of $360 \times 360 \times 180$ stance configurations within a half minute, and thereby plays a key role in selecting and aligning the optimal combination of artificial joint components during surgery.

Future work includes the parallelization of V-COLLIDE initialization in order to obtain further parallel speedup on many CPUs.

Acknowledgments. This work was partly supported by JSPS Grant-in-Aid for Young Scientists (B)(15700030) and Grant-in-Aid for Scientific Research on Priority Areas (16016254 and 16035209) by the Ministry of Education, Culture, Sports, Science and Technology of Japan.

\section{References}

1. DiGioia, A.M., et al.: Image guided navigation system to measure intraoperatively acetabular implant alignment. Clinical Orthopaedics and Related Research 355 (1998) 8-22

2. Jaramaz, B., et al.: Computer assisted measurement of cup placement in total hip replacement. Clinical Orthopaedics and Related Research 354 (1998) 70-81

3. Sato, Y., et al.: Intraoperative simulation and planning using a combined acetabular and femoral (CAF) navigation system for total hip replacement. In: Proc. 3rd Int'l Conf. Medical Image Computing and Computer-Assisted Intervention (MICCAI'00). (2000) 1114-1125

4. Richolt, J.A., et al.: Impingement simulation of the hip in SCFE using 3D models. Computer Aided Surgery 4 (1999) 144-151

5. Kang, M., et al.: Accurate simlation of hip joint range of motion. In: Proc. 15th Int'l Conf. Computer Animation (CA'02). (2002) 215-219

6. Kawasaki, Y., et al.: High-performance computing service over the Internet for intraoperative image processing. IEEE Trans. Information Technology in Biomedicine 8 (2004) 36-46

7. Boden, N.J., et al.: Myrinet: A gigabit-per-second local-area network. IEEE Micro 15 (1995) 29-36

8. O'Carroll, F., et al.: The design and implementation of zero copy MPI using commodity hardware with a high performance network. In: Proc. 12th ACM Int'1 Conf. Supercomputing (ICS'98). (1998) 243-250

9. Message Passing Interface Forum: MPI: A message-passing interface standard. Int'l J. Supercomputer Applications and High Performance Computing 8 (1994) 159-416

10. Gottschalk, S., et al.: OBBTree: A hierarchical structure for rapid interference detection. Computer Graphics (Proc. ACM SIGGRAPH'96) 30 (1996) 171-180

11. Hudson, T.C., et al.: V-COLLIDE: Accelerated collision detection for VRML. In: Proc. 2nd Symp. Virtual Reality Modeling Language (VRML'97). (1997) 117-124

12. Sugano, N., et al.: Accuracy evaluation of surface-based registration methods in a computer navigation system for hip surgery performed through a posterolateral approach. Comput. Aided Surgery 6 (2001) 195-203 